\title{
Type Synthesis of Lower Mobility Parallel Mechanisms: A Review
}

\author{
Wei Ye and Qinchuan Li
}

\begin{abstract}
Type synthesis of mechanisms aims to systematically determine all possible structures for a specific mobility requirement. Numerous methods based on different theories were proposed for type synthesis of lower mobility parallel mechanisms in past decades. However, there does not exist a comprehensive review on these approaches. Therefore, the goal of this paper is to give such a review, classifying the approaches proposed in the literature into three groups, namely, motion-based methods, constraint-based methods, and other methods. The motion-based methods include the Lie group based method, the $G_{F}$ set method, the linear transformation method, the POC set method, and the finite screw method. The constraint-based methods involve the screw theory-based method, the virtual chain method, the method based on Grassmann line geometry and line graphs, and the motion constraint generator method. Other methods contain the enumeration approach based on the general CGK mobility formula and the graph theory method. Upon thoroughly analyzing the characteristics and/or limitations of each method, this review provides a well reference to help researchers find an effective synthesis method for innovative design and further scientific investigations for mechanisms.
\end{abstract}

Keywords: Parallel mechanism, Lower mobility, Type synthesis, Innovative design

\section{Introduction}

According to the definition of IFToMM [1], a parallel mechanism (PM) is a manipulator that controls the motion of its moving platform using at least two kinematic chains going from the moving platform toward the frame. The number of kinematic chains is generally equal to that of the PM's degrees of freedom (DOFs); hence, we can arrange one motor near to the fixed base on each chain to fully actuate the PM. PMs have the advantages of high stiffness, high accuracy, and good dynamic performance compared with their serial counterparts. An amusement device invented in 1931 by Gwinnett [2] may have been the first example of PM. Approximately 30 years later, the most famous 6-DOF PM known as the Gough-Stewart platform, was initially devised as a tire testing device [3], and then widely applied in the field of motion simulation [4]. PMs have gradually drawn the attention of the research community since Hunt

*Correspondence: lqchuan@zstu.edu.cn

Faculty of Mechanical Engineering and Automation, Zhejiang Sci-Tech University, Hangzhou 310018, China published his well-known treatise [5] in which PMs were recommended as robotic mechanisms.

Type synthesis is considered as a major topic in the research of PM. It is defined as innovatively designing topological structures that generate prescribed mobility so as to meet application requirements. Type synthesis is a non-numerical and nonlinear issue. For a prescribed mobility, there exists many possible schemes for designing mechanisms. The benefit is that promising candidates can be obtained from these schemes while considering practical constraints. The term "mobility" has several definitions corresponding to different synthesis approaches, such as the number of DOFs, the motion characteristics (translation and/or rotation), and the sequence of translations and rotations. Information about the resulting structures, including the number of limbs, types of kinematic joints and their geometrical relationships in each limb, and geometrical conditions between different limbs, should be obtained in synthesis procedures.

The type synthesis of 6-DOF PMs is generally easy. A variety of 6-DOF PMs can be constructed by connecting a moving platform to a fixed base through several 6-DOF 
kinematic chains [6-8]. Note that, in many applications, such as pick-and-place, orienting devices, and parallel kinematic machines, PMs with fewer than 6 DOFs, called lower mobility PMs, are sufficient. Lower mobility means fewer kinematic chains and motors, which results in, for example, the advantages of a larger workspace, lower cost, and simpler controller. Therefore, a lower mobility PM that satisfies the mobility requirement of an application is preferable to a 6-DOF PM, which places an urgent demand on new structures of lower mobility PMs. However, the type synthesis of lower mobility PMs is challenging, as stated by Merlet [9]: "This (type synthesis) is a very complex problem for parallel mechanism at the opposite of open loop mechanism for which the number of possible combinations is relatively reduced." The early stage of the construction of lower mobility PMs mainly relied on the experience and inspiration of designers. Typical examples (Figure 1) include the Delta robot [10] with three translational (3T) DOFs, the agile eye [11] with three rotational (3R) DOFs, the 3-RPS PM with two translational and one rotational DOFs [12], and the H4 robot with three translational and one rotational DOFs
[13]. Despite this, the varieties of PMs were far from adequate, and some PMs required for practical applications, such as 5-DOF PMs with identical limbs, were believed to be non-existent.

The investigation on systematic type synthesis approaches of lower mobility PMs dates back to the last century and has experienced a gold period with great progress at the beginning of this century. Based on various mathematical tools, a variety of approaches have been presented in the literature, including the Lie group theory-based method [14-29], the method based on $G_{F}$ (generalized function) sets [30-35], linear transformation method [36-46], position and orientation characteristic (POC) set method [47-52], finite screw method [53-59], screw theory-based method [60-74], virtual chain method [75-79], method based on Grassmann line geometry and line graphs [80-84], motion constraint generator method [85-89], method based on the Chebyshev-Grübler-Kutzbach (CGK) mobility formula [90], and graph theory method [91-93]. According to their synthesis ways, the approaches proposed in the literature can be classified into three categories: motion-based

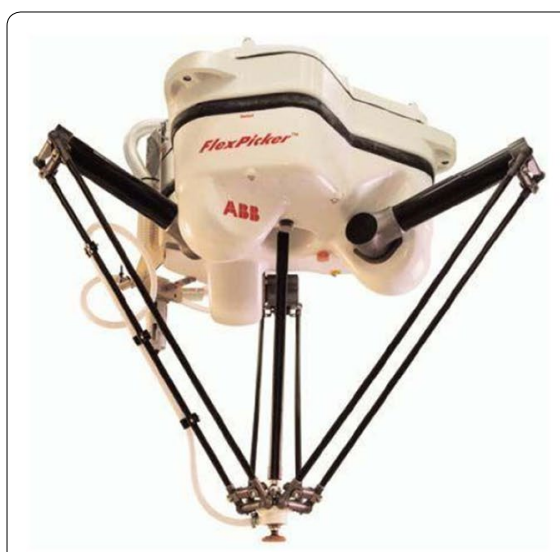

a

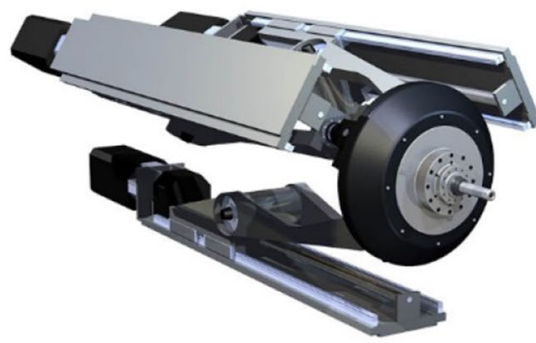

d

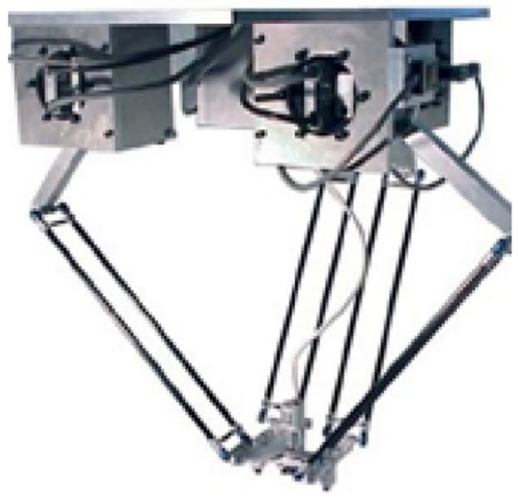

b

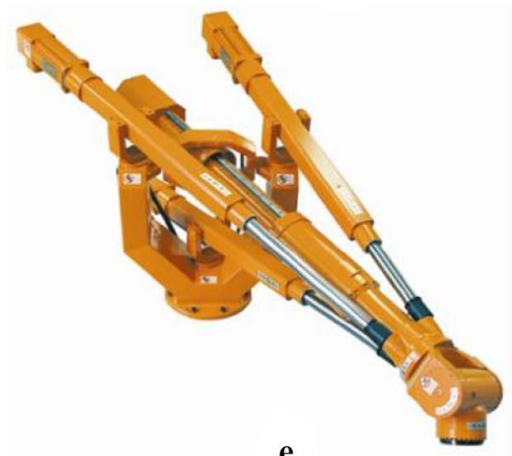

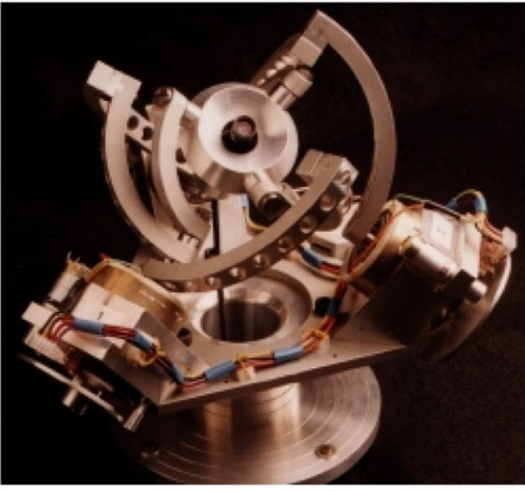

c

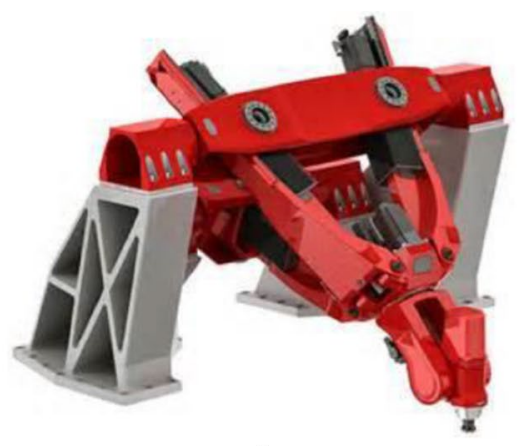

f

Figure 1 Typical lower mobility PMs: a the Delta robot (https://new.abb.com/products/robotics/industrial-robots/irb-360); b the H4 robot [13]; c the agile eye (https://robot.gmc.ulaval.ca/en/research/research-thrusts/parallel-mechanisms/the-agile-eye/); $\mathbf{d}$ the Z3 spindle head (http://www. ds-technologie.de); e the Tricept robot (http://www.pkmtricept.com/); and $\mathbf{f}$ the Exechon robot (http://unisonltd.com/innovation/exechon/) 
methods, constraint-based methods, and other methods. The basic idea of motion-based methods is that the motion of a PM's moving platform is the intersection of those allowed by the parallel kinematic chains that connect the moving platform to the fixed base. Methods in this category, also regarded as direct methods, are represented by the Lie group theory-based method. By contrast, the basic idea of constraint-based methods is that the constraint of a PM's moving platform is the union of those generated by the parallel kinematic chains. This type of method, represented by the screw theory-based method, can be considered as indirect methods since the constraints are taken into account rather than the motions. Methods that cannot be placed in the same category as the above two types are referred to as other methods.

Although the type synthesis of lower mobility PMs has been extensively studied for over 20 years, the research community does not have a comprehensive review on this topic. To the best of our knowledge, the only review on the type synthesis of PMs is [94], which introduced a framework for type synthesis, and then briefly reviewed several methods under that framework. Therefore, this paper provides a comprehensive review on the state-ofthe-art approaches of type synthesis for lower mobility PMs. It aims to enable readers to find corresponding efficient mechanism design methods for different requirements with various characteristics.

\section{Motion-Based Methods}

Generally, motion-based methods use some mathematical expressions, such as a displacement group/manifold, set, and matrix, to represent the motion of joints and bodies, and then establish maps between topological structures and motion using mathematical tools. Methods in this category include the Lie group theory-based method, method based on $G_{F}$ sets, linear transformation method, POC set method, and finite screw method.

\subsection{Lie Group Theory-Based Method}

A publication in 1978 [14] lay the foundation for using Lie group theory in the type synthesis of PMs. In that paper, the 6-DOF motion of a rigid-body was proven to have a group-algebra structure, and can be denoted by a Lie group $\{\boldsymbol{D}\}$. Additionally, 11 types of motion with fewer than 6 DOFs, that is, 0-DOF rigid connection termed $\{\boldsymbol{E}\}, 1-\mathrm{DOF}$ rotation termed $\{\boldsymbol{R}(N, \boldsymbol{u})\}, 1-\mathrm{DOF}$ translation termed $\{\boldsymbol{T}(\boldsymbol{u})\}, 1$-DOF helical motion termed $\{\boldsymbol{H}(N, \boldsymbol{u}, p)\}$, 2-DOF planar translation termed $\{\boldsymbol{T} 2(\boldsymbol{w})\}, 2$-DOF cylindrical motion termed $\{\boldsymbol{C}(N, \boldsymbol{u})\}$, 3-DOF planar gliding motion termed $\{\boldsymbol{G}(\boldsymbol{u})\}$, 3-DOF spatial translation termed $\{\boldsymbol{T}\}$, 3-DOF rotation around a point termed $\{\boldsymbol{S}(N)\}$, 3 -DOF motion including two translations in a plane and a helical motion with its axis perpendicular to that plane termed $\{\boldsymbol{Y}(\boldsymbol{w}, p)\}$, and 4-DOF Schoenflies motion termed $\{\boldsymbol{X}(\boldsymbol{u})\}$, also have the algebraic properties of groups and are subgroups of $\{\boldsymbol{D}\}$. All the aforementioned types of motion can be generated by different lower pairs or combinations of them that are called mechanical generators. Then, Lie group theory was used in the type synthesis of lower mobility PMs, including 3-DOF translational PMs [15] and 3-DOF rotational PMs [16].

At an early stage, applications of the synthesis method based on Lie group theory were limited to PMs that had subgroup-type motion. However, there exist some types of motion that do not have the algebraic properties of groups. These types of motion can be divided into two classes. Motions in the first class are submanifolds of $\{\boldsymbol{D}\}$ and can be expressed by the union of several subgroups, such as the 5-DOF 3R2T motion that can be expressed by the union of two subgroups $\{\boldsymbol{S}(N)\}\{\boldsymbol{G}(\boldsymbol{u})\}$. Motions in the second class are quite complex and cannot be expressed by the union of subgroups. A typical example is the output motion of the 3RPS PM [12]. For PMs that have motion in the first class, the Lie group theory-based method is still effective. 3R2T PMs [17], 1T2R PMs [1821], 3T2R PMs [22], 2R PMs [23], and 2T2R PMs [24, 25] have been synthesized. A general formula used in type synthesis is given by

$$
\{\boldsymbol{M}\}=\bigcap_{i=1}^{n}\left\{\boldsymbol{L}_{i}\right\},
$$

where $\{\boldsymbol{M}\}$ represents the motion set of the moving platform, $\left\{\boldsymbol{L}_{i}\right\}$ the motion set (also called the kinematic bond) allowed by limb $i$, and $n$ the number of limbs. $\{\boldsymbol{M}\}$ and $\left\{\boldsymbol{L}_{i}\right\}$ can be either displacement subgroups or displacement submanifolds.

The type synthesis of PMs using Lie group theory can be briefly summarized as follows. First, the motion set $\{\boldsymbol{M}\}$ that corresponds to the mobility requirement is identified. Then, the appropriate kinematic bonds $\left\{\boldsymbol{L}_{i}\right\}$ that satisfy Eq. (1) are determined, which are used to derive the kinematic chains and geometrical conditions for the desired PMs. Finally, PMs are assembled.

We found that synthesized PMs that use Lie group theory have either subgroup-type motion or submanifold-type motion, and both types can be expressed by the union of several fundamental elements, that is, 1-D subgroups. The difference is that the sequence of fundamental elements that constitute a subgroup is changeable, whereas this is not the case for a submanifold. This may explain why the location of rotation in a planar chain (mechanical generator of a 3-DOF planar subgroup) can vary, but the sequence of two rotational axes of a universal joint in a lower mobility PM cannot be randomly interchanged. We should keep this in mind when 
determining kinematic bonds for lower mobility PMs with submanifold-type motion.

Some composite joints that are neither lower pairs nor higher pairs can also be associated with kinematic bonds. Angeles [26] recalled the fundamental concepts of motion representation and groups of displacements related to rigid bodies, and used the II joint, $\mathrm{II}^{2}$ joint, and $\mathrm{II}^{3}$ joint in the qualitative synthesis of lower mobility PMs. The II joint, also called the hinged parallelogram $[22,27,28]$, is used as a component of primitive Schoenflies motion and double Schoenflies motion generators.

Meng et al. [29] indicated that the Lie group theorybased method explores only the algebraic property but not the differential property of special Euclidean group $\mathrm{SE}(3)$, and the computations of subgroups are typically implemented using brute force at the group level. To model primitive joints and task spaces with subgrouptype and/or submanifold-type motion under a unified framework, they explored both the algebraic and differential properties of $\mathrm{SE}(3)$, and its Lie subgroups and submanifolds, and developed a geometric method for mechanism synthesis, which can be considered as an extension of the Lie group theory-based method.

It is noted that a global coordinate system is usually established in the Lie group theory-based method. This is to simplify expression and calculating of vectors and groups involved. These vectors and groups are actually independent with coordinate systems. Further, the synthesis results including number of limbs, types of kinematic joints and their geometrical relationships in each limb, and geometrical conditions between different limbs are independent with coordinate frames, which means the synthesized results are comprehensive and generalized. Also of note is that the provided PMs examples may have several unnecessary and special geometrical conditions. For example, axes of two adjacent revolute joints with no special relationship in a same link are preferable to be arranged as intersecting and perpendicular for practical reasons such as simplifying D-H parameters.

In summary, the Lie group theory-based method has rigorous mathematical foundation and mainly has two advantages. First, finite mobility is guaranteed. Second, it can be used in PMs with mixed rotations and translations whose sequence is specified. However, this method has a high requirement on mathematical foundations of researchers.

\subsection{Method Based on $G_{F}$ Sets}

Generalized function sets, called $G_{F}$ sets, were introduced by Gao et al. [30-35] to denote the motion characteristics of the moving platforms of robots. A $G_{F}$ set is composed of six elements:

$$
G_{F}\left(T_{a} T_{b} T_{c} ; R_{\alpha} R_{\beta} R_{\gamma}\right),
$$

where the first three and last three elements represent the translational and rotational characteristics of the moving platform, respectively. The six elements can be either specific symbols or zero, which signify the existence or non-existence, respectively, of the corresponding characteristics. Therefore, different types of motion can be denoted by different $G_{F}$ sets. Intersection laws associated with translational characteristics and rotational characteristics have been established, which lay the foundation for intersection operations between $G_{F}$ sets, and thus provide an effective and general method for the type synthesis of PMs. This method can be expressed by a general formula as

$$
G_{F}=G_{F 1} \cap G_{F 2} \cap \cdots \cap G_{F n},
$$

where $G_{F}$ denotes the motion set of the moving platform and $G_{F i}(i=1, \ldots, n)$ represents the motion set of limb $i$.

There is a total of 21 types of $G_{F}$ sets, which are classified into two classes according to the sequence of translational characteristics and rotational characteristics, that is, first-class $G_{F}$ sets and second-class $G_{F}$ sets, respectively. The type synthesis of PMs that have the motion of first [31,32] and second-class $G_{F}$ sets [33] is conducted successively.

The axis movement theorems established in the $G_{F}$ set method indicate that if the axis of a revolute joint is always perpendicular to the 2-DOF translational characteristics within the same limb, then the location variation of the revolute joint does not affect the motion characteristics of that limb, whereas if a limb has 3-DOF translational characteristics, then its revolute joints can be arbitrarily arranged without affecting limb motion. These theorems can be explained easily using the closures of subgroup product in Lie group theory: a planar subgroup $\{\boldsymbol{G}(\boldsymbol{u})\}$ or Schoenflies subgroup $\{\boldsymbol{X}(\boldsymbol{u})\}$ allows rotation around axis $\boldsymbol{u}$ in any location.

Type synthesis based on $G_{F}$ sets has a similar procedure as that using Lie group theory. The motion of the desired PMs is expressed using $G_{F}$ sets first. Then, $G_{F i}$ that satisfies Eq. (3) is determined following some intersection laws [33]. Finally, kinematic limbs that correspond to $G_{F i}$ are obtained and used to construct PMs. This method results in PMs with finite mobility and is applicable to PMs with both rotations and translations.

\subsection{Linear Transformation Method}

A mechanism can generate a set of output velocities given an appropriate set of active joint velocities. It is actually a linear transformation from the joint velocity space to the output velocity space, and this transformation is represented by the Jacobian matrix. According to the differences in Jacobian matrices, PMs can be classified into five types [36]: (i) maximally regular PMs whose Jacobian 
matrices are identity matrices throughout the workspace; (ii) fully isotropic PMs whose Jacobian matrices are diagonal matrices with identical elements throughout the workspace; (iii) PMs with uncoupled motion whose Jacobian matrices are diagonal matrices with different diagonal elements; (iv) PMs with decoupled motion whose Jacobian matrices are triangular matrices; and (v) PMs with coupled motion whose Jacobian matrices are general matrices.

Based on the theory of linear transformations, Gogu [36-46] presented a new type synthesis method for lower mobility PMs. This method first identifies some conditions that correspond to the desired output motion, that is, general conditions with no actuator locked and particular conditions with one actuator locked. These conditions ensure a one-to-one mapping between output velocities and active joint velocities. Then limb structures are designed that obey those conditions, which can be conducted using an evolutionary morphology approach [36, 37]. Finally, PMs can be constructed using an appropriate number of limbs. This method is suitable for the type synthesis of PMs that have special mappings between joint velocity space and output velocity space, which means it can be used to synthesize isotropic PMs and maximally regular PMs. It has been applied to the type synthesis of fully isotropic translational PMs [38], fully isotropic 2-DOF parallel wrists [39], fully isotropic 3-DOF parallel wrists [40], fully isotropic PMs with Schoenflies motion [37, 41], fully isotropic planar PMs [42], fully isotropic T1R2 PMs [43], fully isotropic T2R2 PMs [44], fully isotropic T3R2 PMs [45], maximally regular T3R2 PMs [36], and maximally regular planar PMs [46].

\subsection{POC Set Method}

Yang et al. [47-52] indicated that a topological structure generally has three essential features, including kinematic pair types, dimensional constraint types, and connections between structure units. Six basic dimensional constraint types that describe geometrical conditions between kinematic pairs were presented, such as coincidence of joint axes and perpendicularity of joint axes. The three essential features help to establish symbol representations of mechanism structures. Then, the authors proposed representing the output motion of mechanisms using POC sets, which have a matrix form as follows:

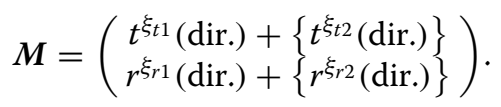

Translation elements and rotation elements of the output motion are distinguished in the POC matrix $M$ with $t^{\xi t i}$ (dir.) representing the former and $r^{\xi_{r i}}$ (dir.) representing the latter. The motion of kinematic pairs, dimensional constraint types, and moving platforms of PMs can be expressed by POC matrices. Then, a POC equation can be established for a PM as

$$
\boldsymbol{M}_{\mathrm{Pa}}=\stackrel{(v+1)}{\bigcap} \boldsymbol{M}_{b i},
$$

where $\boldsymbol{M}_{b i}$ is the POC matrix of limb $i, \boldsymbol{M}_{\mathrm{Pa}}$ represents the POC matrix of the moving platform, and $v$ is the number of independent loops in the PM.

Several operation rules, including the linear operation among rotation elements, linear operation among translation elements, and nonlinear criteria for determining independent elements, were proposed for Eq. (5), which lay the foundation for structural analysis (forward operation of the POC equation), in addition to type synthesis (inverse operation of the POC equation) of PMs using the method of POC sets.

The POC method can be used in type synthesis of PMs with both rotations and translations. It has been used in the type synthesis of 3-DOF translational PMs [48], 1T2R partially decoupled PMs [49], 3T1R PMs [50], and some other lower mobility PMs $[51,52]$.

\subsection{Finite Screw Method}

Derived from the expression of Chasles' motion with a unit dual quaternion [53], Sun et al. [54] presented another approach to describe motion using a finite screw as

$$
\boldsymbol{S}_{f}=2 \tan \frac{\theta}{2}\left(\begin{array}{c}
\boldsymbol{s}_{f} \\
\boldsymbol{r}_{f} \times \boldsymbol{s}_{f}
\end{array}\right)+t\left(\begin{array}{c}
\mathbf{0} \\
\boldsymbol{s}_{f}
\end{array}\right),
$$

where $s_{f}$ is a unit vector that represents the axis of the finite screw, $\boldsymbol{r}_{f}$ is a position vector from the origin of a reference frame to an arbitrary point on the screw axis, and $\theta$ and $t$ represent rotational and translational displacements, respectively.

For a serial limb, the finite motion of its end link is the composition of those generated by all the joints in that limb. Accordingly, the finite screw expression of the limb motion can be represented by the screw triangle product [55] of the finite screws of the joints. Whereas for a PM, the finite screw of its moving platform can be obtained as the intersection of finite screws of all the limbs. Under the guidance of these principles, a synthesis method based on the finite screw has been proposed and applied to the type synthesis of 3T PMs [56], 3T1R PMs with a variable rotational axis [57], 2T3R PMs [58], and 1T2R PMs [59]. This method mainly has two features. First, the synthesized PMs have full cycle mobility. Second, the involved mathematical operations are complex and requires good mathematical foundations. 


\section{Constraint-Based Methods}

For a mechanism, its mobility and constraints are two opposite concepts. Constraints can be physically described as constraint forces or constraint couples, which restrict translational DOFs and rotational DOFs, respectively. If a spatial mechanism has 6 DOFs, then there is no constraint on its moving platform, and if a mechanism has fewer than 6 DOFs, then there must be some constraints exerted on its moving platform. Let the number of DOFs of a mechanism be denoted by $F$ and the number of independent constraints by $C$, then a general formula can be given as

$$
F=6-C \text {. }
$$

For a lower mobility PM, its number of DOFs is fewer than six. There is a one-to-one mapping between the DOFs and constraints of its moving platform. For example, there must be a constraint force exerted on the moving platform of a 3R2T PM, and three constraint couples should be generated for a 3T PM. This type of mapping means that we can design PMs with the desired mobility from the perspective of constraints because some mathematical tools manage constraints well. Therefore, a class of constraint-based methods, which can be regarded as indirect methods, has been proposed for the type synthesis of lower mobility PMs.

\subsection{Screw Theory-Based Method}

Research on screw theory dates back to the 18th century [53]. Poinsot proved that the force system of a rigid body can be reduced to a wrench that includes a force along a straight line and a moment around that line. Chasles indicated that the transformation of a rigid body from one position to another is equal to a combined motion (termed twist) that includes a translation along a line and a rotation around that line. These two important discoveries established the foundation of screw theory. Then, Ball [60] presented the concept of the screw system, and proved the reciprocity between a twist system and wrench system, which provides a mathematical mapping of the motion and constraints of rigid bodies. A unit screw can be defined by two vectors as

$$
\boldsymbol{S}=\left(\boldsymbol{s} ; \quad \boldsymbol{s}_{\boldsymbol{o}}\right)=(\boldsymbol{s} ; \quad \boldsymbol{r} \times \boldsymbol{s}+p \boldsymbol{s}),
$$

where unit vector $s$ represents the axis direction of the screw, $\boldsymbol{r}$ is a position vector of an arbitrary point on the axis of the screw with respect to a reference frame, and $p$ represents the pitch of the screw. This screw can represent a general motion (twist) or general force (wrench). If pitch $p$ equals zero, then the screw presents a rotation or force, whereas a translation or couple can be represented by a screw $(\boldsymbol{0} ; \boldsymbol{s})$. Screw $\boldsymbol{\phi}^{\boldsymbol{r}}=\left(\boldsymbol{s} ; \quad \boldsymbol{s}_{\boldsymbol{o r}}\right)$ is reciprocal to screw system $\left\{\boldsymbol{\phi}_{1}, \boldsymbol{S}_{2}, \ldots, \boldsymbol{S}_{n}\right\}$ if the following condition is satisfied:

$$
\boldsymbol{S}^{\boldsymbol{r}} \circ \boldsymbol{\phi}_{j}=\boldsymbol{s}_{\boldsymbol{o r}} \cdot \boldsymbol{s}_{j}+\boldsymbol{s}_{\boldsymbol{o} j} \cdot \boldsymbol{s}_{\boldsymbol{r}}=0,(j=1,2, \ldots, n) .
$$

After knowing the natural advantage of screw theory in terms of representing the motion and forces of rigid bodies, researchers in the community started to promote this theory, and made significant contributions to its applications in mechanisms and robots [53]. Regarding type synthesis, Huang [61-65] first proposed a constraint synthesis method based on screw theory, which can solve the problem of synthesizing symmetrical lower mobility PMs. Fang et al. [66-69] and Kong et al. [70-74] also synthesized many lower mobility PMs using screw theory, such as 3-DOF PMs [67, 68, 70, 71, 73], 4-DOF PMs [66, 69, 72], and 5-DOF PMs [66].

The procedures of this method can be summarized as follows:

(i) Express the constraints of PMs that have the desired mobility using wrench system $W$.

(ii) Identify the number of limbs (typically equals the number of DOFs of the PMs). Then determine the wrench system of each limb following the condition that the union of limb wrench systems should equal $W$.

(iii) Calculate the twist system for each limb following Eq. (9). Then apply a linear combination in each system to obtain a general twist, which is used to identify the joint types and geometrical conditions in each limb.

(iv) Assemble PMs using the synthesized limb structures, in which some assembly conditions may be required to ensure that the union of the limb wrench systems is $W$.

(v) Conduct a mobility check to ensure that the PMs have full cycle mobility throughout their workspaces, except singular configurations.

A screw describes the motion of a rigid body at the velocity level, which means the motion of a screw is instantaneous. Therefore, further checks regarding full cycle mobility should be conducted for the synthesized PMs as in step (v), which can be finished using a simple, rigorous, and generic method [95].

It is noted that there are some confusions about the limb coordinate frame and the global coordinate frame in Huang's method [61-65]. A coordinate frame is attached to a kinematic limb to express and calculate joint twists and their reciprocal constraint screws. Then the constraint screws are interpreted as lines in space based on their geometric nature. Because these lines are coordinate-free their linear dependence can be identified by investigating their geometrical conditions in the PMs. In other words, there is a subtle manipulation between 
coordinate-dependent algebraic expression and coordinate-free geometric meaning.

It should be also noted that the established reference systems will facilitate the subsequent analysis about kinematics and dynamics. The screw theory-based method is comprehensive, generalized, and has rigorous mathematical foundation, which is applicable to PMs with mixed rotations and translations.

\subsection{Virtual Chain Method}

Generally, the motion pattern of a lower mobility PM can be described using several kinematic chains, among which the simplest is called the virtual chain of that PM [75]. The virtual chain indicates clearly the motion pattern of the associated PM, and they have the same wrench system. The virtual chain method was presented by Kong et al. [75-78] for the type synthesis of lower mobility PMs. This method can be implemented in four steps once we have determined the motion pattern and virtual chain of the desired PMs:

(i) Decompose the wrench system (denoted by $W$ ) of the virtual chain (desired PMs) into several possible subsystems.

(ii) Conduct the type synthesis of single-loop chains with wrench system $W$ using one virtual chain and one other chain. Feasible limb structures can thus be obtained by removing the virtual chain from the single-loop chains.

(iii) Construct PMs with an appropriate number of obtained limbs following the condition that the linear combination of the limb wrench systems should equal $W$.

(iv) Select input joints for the PMs.

The virtual chain method originated from the screwbased method because screw theory plays an important role in the four steps. Features of the virtual chain method include: (i) the motion pattern of the desired PMs is described using a virtual chain, which means that the sequence of rotations and translations is clear; (ii) few derivations are involved; and (iii) it is easy to understand because single-loop chains are constructed prior to PMs. This method has been applied to synthesize PPR-equivalent PMs [75], US-equivalent PMs [76], UP-equivalent PMs [77], and SP-equivalent PMs [78].

It should be noted that the motion equivalent chain method [79] is actually a modified version of the virtual chain method. The only difference is that the former uses some single-loop chains to construct closed-loop chains, which results in PMs with closed-loop subchains. Strictly speaking, these mechanisms should be called hybrid mechanisms.
Since the synthesized PMs have equivalent motion with the virtual chain, the sequence of rotations and translations in the PMs is clear.

\subsection{Method Based on Grassmann Line Geometry and Line Graphs}

Integrating Grassmann line geometry and a line graph method, Xie et al. [80-84] presented a visible synthesis method. In this method, motion and constraints are denoted by line vectors and line graphs. The mutual conversion of the motion line graphs and constraint line graphs is achieved following the Blanding dual rule and its generalized rule. Therefore, the type synthesis of PMs can be conducted in an intuitive manner. Because the tools used are concise (Grassmann line geometry) and visible (line graph), this method can be understood and used by more researchers. It has been used in the type synthesis of 3-DOF PMs [80-82] and 4-DOF PMs [83, 84].

Because line vectors and line graphs are, in fact, special screws and screw systems, the method based on Grassmann line geometry and line graphs can be regarded as a visualized version of the screw theory-based method. An evidence is that the generalized Blanding rule fits well with the derived relationships between constraints and joint axes using screw theory. The most notable feature of this method is its visualization with the help of line graphs. In other words, it can be regarded as a visualized version of the screw-based method.

\subsection{Motion Constraint Generator Method}

To design a lower mobility PM whose moving platform has some constraints, a simple and intuitive approach is to use a single limb that generates all the required constraints and several 6-DOF limbs. The constraint limb, also called a motion constraint generator [85], can be either actuated or passive. At the end of the last century, this method was used to design lower mobility PMs [8689], among which the 2R1T 3-DOF Tricept robot that has a PU limb (motion constraint generator) and three 6-DOF limbs is a typical example. Later, Kuo and Dai [85] presented synthesis procedures to design lower mobility PMs that have such features, thereby forming a systematic type synthesis method using a motion constraint generator. The key step of this method is to identify motion constraint generators that have the same motion characteristic as the desired PMs. Mobility of the synthesized PMs can be directly obtained.

\section{Other Methods}

In addition to motion-based methods and constraintbased methods, other synthesis methods include the enumeration approach based on the general CGK mobility 
formula and the graph theory method. These methods simultaneously consider the DOFs and constraints, and therefore, are different from the former two types of methods.

\subsection{Enumeration Approach Based on the General CGK Mobility Formula}

The CGK formula is well known as a formula for mobility calculation. However, it can also be used in type synthesis because type synthesis can be regarded as an inversion of mobility analysis. The number of limbs and joints in each limb can be calculated using the CGK formula given the DOFs of desired PMs. This is a simple problem, particularly when some special conditions are prescribed, for example, the number of limbs is equal to the PMs' DOFs and the PMs have identical limb structures. Using this method, Tsai [90] enumerated a class of 3-DOF PMs. This method is straightforward and easy to implement, but its disadvantages are obvious: (i) we cannot synthesize PMs that have specific motion characteristics because the CGK formula only concerns the number of DOFs; (ii) it results in only non-over constrained PMs because the GCK formula does not consider redundant constraints; (iii) geometrical conditions between joints in each limb and those between parallel limbs that have a crucial influence on mobility of PMs cannot be derived; and (iv) full cycle mobility cannot be guaranteed because instantaneous constraints are used in the CGK formula. As summarized by Merlet [9], its use is quite simple, but this formula does not take into account the geometry of the arrangement of the kinematic pairs and hence may lead to invalid results.

\subsection{Graph Theory Method}

As a classical theory used in topological analysis and the synthesis of mechanisms, graph theory has been used in the type synthesis of PMs [91], such as planar 3-DOF PMs [92] and spatial 4-DOF PMs [93]. The graph theory synthesis method generally has four steps as follows:

(i) Determine the number of links, including binary links, ternary links, and quaternary links, according to the desired number of DOFs. Many linkage schemes that correspond to a different number of closed loops are obtained and are typically summarized in a table in this step.

(ii) Form topology embryonic graphs (TEG), also called contracted graphs, which can reflect the number of links (except binary links) and closed loops. It should be noted that more than one TEG may be obtained for the same linkage scheme.

(iii) Distribute binary links over the paths in TEGs to derive topological graphs (TGs). In this step, several necessary conditions must be satisfied to avoid some unwelcome scenarios, such as local structures. Similarly, various TGs can be derived from one TEG. However, some TGs may be isomorphic or invalid, which requires a procedure to identify and eliminate them to avoid repeatable and unreasonable mechanisms.

(iv) Replace dots in TGs that have parallel structures with 1-DOF joints (revolute joints or prismatic joints) to derive the PMs. Many PMs can be synthesized from one TG because different combinations of joints can be applied to that TG.

The graph theory synthesis method is intuitive because of its visualization, and it is convenient to implement with the help of computer programs. However, it also has some limitations. First, it cannot synthesize PMs with specific motion characteristics because only the number of DOFs is of concern in the first step. Second, this method is not very suitable for spatial mechanisms because joint axes that have an important influence on the mobility of spatial mechanisms are not considered. This problem is not so serious in planar mechanisms because their geometrical conditions of joint axes are much simpler than those in spatial mechanisms. Third, this method has a problem regarding synthesizing overconstrained mechanisms because constraints in spatial mechanisms are highly dependent on geometrical conditions of joint axes. Fourth, TGs that result in PMs are a small part of all available TGs. Most TGs generate multiloop complex mechanisms. Determining TGs with parallel structures currently relies on human inspection.

\section{Discussions}

The above methods have made significant achievements in type synthesis of lower mobility PMs. Some PMs that were believed to be non-existent, such as 5-DOF PMs with identical limbs were obtained successfully, which has enriched the varieties of PMs and accelerated the development of robotics and mechanisms. In addition, a lot of PMs with practical values are invented.

In type synthesis, a large number of PMs satisfying a same mobility requirement can be generated. Some constraints are typically considered to limit the resulting candidates. These constraints may include payload capability, workspace, or cost. For instance, to achieve high payload capability, we can specify that the first joint of the adopted limbs should be a prismatic joint so that the PMs can be equipped with heavy powerful servomotors. The selection of reasonable constraints is important because it helps us to quickly determine appropriate candidates for the task and exclude many inappropriate ones. Constraints associated with practical applications should be of particular concern. Indeed, in most cases, 
type synthesis is carried out to design mechanisms compatible with practical tasks. As a thumb of rule, it is better to design a limb with less than three joints and two links, and require no strict assembly conditions. The type synthesis of PMs considering special conditions has been recently conducted to invent some performance-specified or application-orientated PMs, such as PMs without parasitic motion [18, 25], fully isotropic PMs [38-45] and maximally regular PMs [36, 46], PMs with a variable rotational axis [57], PMs without intersecting axes $[96,97]$, orthogonal translational PMs $[98,99]$, non-overconstrained PMs $[69,100]$, and PMs with high rotational capability [21, 101-103]. This kind of type syntheses is very meaningful because it generates PMs that satisfy not only a mobility requirement but also some other special requirements. Nevertheless, we should seriously consider which method is appropriate for the task, otherwise desired results will not be generated.

Regarding the future directions of type synthesis, there are three suggestions: (1) Type synthesis of lower-mobility PMs should take structural indices into consideration. This kind of indices mainly depends on the number of links and joints, etc., which reflects the practical value of mechanisms. However, these practical values are ignored in previous researches in the literature; (2) There are usually some constraints applied in the synthesis that limit the generated PMs in a narrow scope. It would be meaningful to combine the synthesis methods by using artificial intelligence techniques to obtain more candidates; (3) Novel synthesis methods that are applicable to generalized PMs should be further studied. Existing methods were tailored for the design of PMs with one moving platform connected to a fixed base through parallel limbs. It remains challenging to invent mechanisms incorporating more than one moving platform or hybrid limbs.

\section{Conclusions}

(1) The type synthesis of lower mobility PMs has been widely studied for over 20 years. A variety of synthesis methods based on different theories have been presented successively. This paper presented a comprehensive overview of the literature related to this topic, and classified the existing methods into three groups. Fundamental ideas of each method, together with their characteristics and/or limitations were summarized. Several discussions about constraint consideration in type synthesis were presented.

(2) The aim of this paper is to help researches to gain an overall understanding of the state-of-the-art type synthesis approaches of lower-mobility parallel mechanisms, and provide suggestions when selecting appropriate methods.

\section{Authors' Contributions}

QL was in charge of the whole trial; WY wrote the manuscript. Both authors read and approved the final manuscript.

\section{Authors' Information}

Wei Ye, born in 1988, is currently a lecturer at Zhejiang Sci-Tech University, China. He received his PhD degree on mechanism design and theory from Beijing Jiaotong University, China, in 2016. His research interests include design and analysis of reconfigurable parallel mechanisms.

Qinchuan Li, born in 1975, is currently a professor at Zhejiang Sci-Tech University, China. He received his PhD degree on mechanism design and theory from Yanshan University, China, in 2003. His research interests include mechanism theory of parallel manipulators and application.

\section{Competing interests}

The authors declare that they have no competing interests.

\section{Funding}

Supported by National Natural Science Foundation of China (Grant Nos. $51705465,51525504)$, and Young Researchers Foundation of Zhejiang Provincial Top Key Academic Discipline of Mechanical Engineering of Zhejiang Sci-tech University (Grant No. ZSTUME02B02).

Received: 14 August 2018 Accepted: 2 April 2019

Published online: 24 April 2019

References

[1] IFToMM. IFToMM terminology/English 5.1. Mechanism and Machine Theory, 2003, 38(7-10): 819-825.

[2] J E Gwinnett. Amusement devices: US, 1789680. 1928-10-01[1931-0120]. https://patents.glgoo.top/patent/US1789680A/en.

[3] V Gough, S Whitehall. Universal tyre test machine. Proceedings of the 9th International Technical Congress, London, UK, April 30-May 5, 1962: 117-137.

[4] D Stewart. A platform with six degrees of freedom. Proceedings of the Institution of Mechanical Engineers, 1965, 180(1): 371-386.

[5] K H Hunt. Kinematic geometry of mechanisms. New York: Oxford University Press, 1978.

[6] R Alizade, C Bayram. Kinematic and dynamic analysis of a new type of spatial 6-DOF parallel structure manipulator. Proceedings of the 11th World Congress on Mechanism and Machine Science, Tianjin, China, August 18-21, 2003: 1-5.

[7] N Mouly, J P Merlet. Singular configurations and direct kinematics of a new parallel manipulator. Proceedings of the IEEE International Conference on Robotics and Automation, Nice, France, May 12-14, 1992: 338-343.

[8] R I Alizade, N R Tagiyev, J Duffy. A forward and reverse displacement analysis of a 6-DOF in-parallel manipulator. Mechanism and Machine Theory, 1994, 29(1): 115-124.

[9] J P Merlet. Still a long way to go on the road for parallel mechanisms. Proceedings of the ASME International Mechanical Engineering Congress and Exhibition, Montreal, Canada, September 29-October 2, 2002: 95-99.

[10] R Clavel. Delta, a fast robot with parallel geometry. Proceedings of the 18th International Symposium on Industrial Robots, Lausanne, Switzerland, April 26-28, 1988: 91-100.

[11] C M Gosselin, J F Hamel. The agile eye: a high-performance threedegree-of-freedom camera-orienting device. Proceedings of the 1994 IEEE International Conference on Robotics and Automation, San Diego, USA, May 8-13, 1994: 781-786.

[12] KH Hunt. Structural kinematics of in-parallel-actuated robot-arms. Journal of Mechanisms, Transmissions, and Automation in Design, 1983, 105(4): 705-712.

[13] F Pierrot, O Company, S Krut, et al. Four-DOF PKM with articulated travelling-plate. Proceedings of the Parallel Kinematics Seminar, Chemnitz, Germany, April 25-26, 2006: 25-26. 
[14] J M Hervé. Analyse structurelle des mécanismes par groupe des déplacements. Mechanism and Machine Theory, 1978, 13(4): 437-450.

[15] J M Hervé. The Lie group of rigid body displacements, a fundamental tool for mechanism design. Mechanism and Machine Theory, 1999, 34(5): 719-730.

[16] M Karouia, J M Hervé. A three-DOF tripod for generating spherical rotation. In: J Lennarčič, M M Stanišić. Advances in robot kinematics. Dordrecht: Springer, 2000: 395-402.

[17] Q C Li, Z Huang, J M Hervé. Type synthesis of 3R2T 5-DOF parallel mechanisms using the Lie group of displacements. IEEE Transactions on Robotics and Automation, 2004, 20(2): 173-180.

[18] Q C Li, J M Hervé. 1T2R parallel mechanisms without parasitic motion. IEEE Transactions on Robotics, 2010, 26(3): 401-410.

[19] Q C Li, J M Hervé. Type synthesis of 3-DOF RPR-equivalent parallel mechanisms. IEEE Transactions on Robotics, 2014, 30(6): 1333-1343.

[20] Q C Li, L M Xu, Q H Chen, et al. New family of RPR-equivalent parallel mechanisms: design and application. Chinese Journal of Mechanical Engineering, 2017, 30(2): 217-221.

[21] WYe, Q C Li, XX Chai. New family of 3-DOF UP-equivalent parallel mechanisms with high rotational capability. Chinese Journal of Mechanical Engineering, 2018, 31(1): 12

[22] C C Lee, J M Hervé. Generators of the product of two Schoenflies motion groups. European Journal of Mechanics-A/Solids, 2010, 29(1): 97-108.

[23] J M Hervé. Uncoupled actuation of pan-tilt wrists. IEEE Transactions on Robotics, 2006, 22(1): 56-64.

[24] C X Fan, H Z Liu, Y B Zhang. Type synthesis of 2T2R, 1T2R and 2R parallel mechanisms. Mechanism and Machine Theory, 2013, 61: 184-190.

[25] WYe, LY He, Q C Li. A new family of symmetrical 2T2R parallel mechanisms without parasitic motion. Journal of Mechanisms and Robotics, 2018, 10(1): 011006

[26] J Angeles. The qualitative synthesis of parallel manipulators. Journal of Mechanical Design, 2004, 126(4): 617-624.

[27] C C Lee, J M Hervé. Type synthesis of primitive Schoenflies-motion generators. Mechanism and Machine theory, 2009, 44(10): 1980-1997.

[28] C C Lee, J M Hervé. On some applications of primitive Schönfliesmotion generators. Mechanism and Machine Theory, 2009, 44(12): 2153-2163.

[29] J Meng, G F Liu, Z X Li. A geometric theory for analysis and synthesis of sub-6 DOF parallel manipulators. IEEE Transactions on Robotics, 2007, 23(4): 625-649.

[30] F Gao, W M Li, X C Zhao, et al. New kinematic structures for 2-, 3- 4and 5-DOF parallel manipulator designs. Mechanism and Machine Theory, 2002, 37(11): 1395-1411.

[31] J L Yang, F Gao, Q J Ge, et al. Type synthesis of parallel mechanisms having the first class GF sets and one-dimensional rotation. Robotica, 2011, 29(6): 895-902.

[32] J L Yang, F Gao, K J Zhu, et al. Type synthesis of parallel mechanisms with the first class GF sets and two-dimensional rotations. International Journal of Advanced Robotic Systems, 2012, 9(3): 61.

[33] F Gao, J L Yang, Q J Ge. Type synthesis of parallel mechanisms having the second class GF sets and two dimensional rotations. Journal of Mechanisms and Robotics, 2011, 3(1): 011003.

[34] J He, F Gao, X D Meng, et al. Type synthesis for 4-DOF parallel press mechanism using GF set theory. Chinese Journal of Mechanical Engineering, 2015, 28(4): 851-859.

[35] X D Meng, F Gao. The classification of GF sets for robotic mechanisms. Proceedings of the 14th IFTOMM World Congress, Taipei, China, October 25-30, 2015: 145-153.

[36] G Gogu. Structural synthesis of maximally regular T3R2-type parallel robots via theory of linear transformations and evolutionary morphology. Robotica, 2009, 27(1): 79-101.

[37] G Gogu. Structural synthesis of fully-isotropic parallel robots with Schönflies motions via theory of linear transformations and evolutionary morphology. European Journal of Mechanics-A/Solids, 2007, 26(2): 242-269.

[38] G Gogu. Structural synthesis of fully-isotropic translational parallel robots via theory of linear transformations. European Journal of Mechanics-A/Solids, 2004, 23(6): 1021-1039.

[39] G Gogu. Fully-isotropic over-constrained parallel wrists with two degrees of freedom. Proceedings of the 2005 IEEE International Conference on Robotics and Automation, Barcelona, Spain, April 18-22, 2005: 4014-4019.

[40] G Gogu. Fully-isotropic three-degree-of-freedom parallel wrists. Proceedings of the 2007 IEEE International Conference on Robotics and Automation, Roma, Italy, April 10-14, 2007: 895-900.

[41] G Gogu. Singularity-free fully-isotropic parallel manipulators with Schonflies motions. Proceedings of the 12th IEEE International Conference on Advanced Robotics, Seattle, USA, July 18-20, 2005: 194-201.

[42] G Gogu. Fully-isotropic over-constrained planar parallel manipulators. Proceedings of the 2004 IEEE/RSJ International Conference on Intelligent Robots and Systems, Sendai, Japan, September 28-October 2, 2004: 3519-3524.

[43] G Gogu. Fully-isotropic T1R2-type parallel robots with three degrees of freedom. Proceedings of the ASME 2005 International Design Engineering Technical Conferences and Computers and Information in Engineering Conference, Long Beach, USA, September 24-28, 2005: 757-764.

[44] G Gogu. Fully-isotropic parallel robots with four degrees of freedom T2R2-type. Proceedings of the 2005 IEEE/RSJ International Conference on Intelligent Robots and Systems, Edmonton, Canada, August 2-6, 2005: 960-965.

[45] G Gogu. Fully-isotropic T3R2-type parallel robotic manipulators. Proceedings of the 2006 IEEE Conference on Robotics, Automation and Mechatronics, Bangkok, Thailand, June 1-3, 2006: 1-6.

[46] G Gogu. Kinematic criteria for structural synthesis of maximally regular parallel robots with planar motion of the moving platform. In: A Kecskeméthy, V Potkonjak, A Müller. Interdisciplinary applications of kinematics, Dordrecht: Springer, 2012: 63-81.

[47] T L Yang, A X Liu, Q Jin, et al. Position and orientation characteristic equation for topological design of robot mechanisms. Journal of Mechanical Design, 2009, 131(2): 021001.

[48] Q Jin, T L Yang. Theory for topology synthesis of parallel manipulators and its application to three-dimension-translation parallel manipulators. Journal of Mechanical Design, 2004, 126(4): 625-639.

[49] Q Jin, T L Yang. Synthesis and analysis of a group of 3-degree-of-freedom partially decoupled parallel manipulators. Journal of Mechanical Design, 2004, 126(2): 301-306

[50] T L Yang, A X Liu, P H Shen, et al. Topological structure synthesis of 3T1R parallel mechanism based on POC equations. Proceedings of the International Conference on Intelligent Robotics and Applications. Tokyo, Japan, August 16-18, 2016: 147-161.

[51] T L Yang. Topology structure design of robot mechanisms. Beijing: China Machine Press, 2004. (in Chinese)

[52] TL Yang, A X Liu, P H Shen, et al. Topology design of robot mechanisms. Singapore: Springer Singapore, 2018.

[53] J S Dai. Geometrical foundations and screw algebra for mechanisms and robotics. Beijing: Higher Education Press, 2014. (in Chinese)

[54] T Sun, S F Yang, T Huang, et al. A way of relating instantaneous and finite screws based on the screw triangle product. Mechanism and Machine Theory, 2017, 108: 75-82.

[55] D Zarrouk, M Shoham. A note on the screw triangle. Journal of Mechanisms and Robotics, 2011, 3(1): 014502.

[56] S F Yang, T Sun, T Huang, et al. A finite screw approach to type synthesis of three-DOF translational parallel mechanisms. Mechanism and Machine Theory, 2016, 104: 405-419.

[57] S F Yang, T Sun, T Huang. Type synthesis of parallel mechanisms having 3T1R motion with variable rotational axis. Mechanism and Machine Theory, 2017, 109: 220-230.

[58] T Sun, S F Yang, T Huang, et al. A finite and instantaneous screw based approach for topology design and kinematic analysis of 5-axis paralle kinematic machines. Chinese Journal of Mechanical Engineering, 2018, $31(2): 44$

[59] T Sun, X Huo. Type synthesis of 1T2R parallel mechanisms with parasitic motions. Mechanism and Machine Theory, 2018, 128: 412-428.

[60] R S Ball. A treatise on the theory of screws. Cambridge: Cambridge University Press, 1900

[61] Z Huang, Q C Li. General methodology for type synthesis of symmetrical lower-mobility parallel manipulators and several novel manipulators. The International Journal of Robotics Research, 2002, 21(2): 131-145.

[62] Z Huang, Q C Li. Type synthesis principle of minor-mobility parallel manipulators. Science in China Series E: Technological Sciences, 2002, 45(3): 241-248. 
[63] Z Huang, Q C Li. Type synthesis of symmetrical lower-mobility parallel mechanisms using the constraint-synthesis method. The International Journal of Robotics Research, 2003, 22(1): 59-79.

[64] Q C Li, Z Huang. Type synthesis of 4-DOF parallel manipulators. Proceedings of the 2003 IEEE International Conference on Robotics and Automation. Taipei, China, September 14-19, 2003, 1: 755-760.

[65] DX Zeng, Z Huang. Type synthesis of the rotational decoupled parallel mechanism based on screw theory. Science China Technological Sciences, 2011, 54(4): 998-1004.

[66] Y F Fang, L W Tsai. Structure synthesis of a class of 4-DoF and 5-DoF parallel manipulators with identical limb structures. The International Journal of Robotics Research, 2002, 21(9): 799-810.

[67] Y F Fang, L W Tsai. Structure synthesis of a class of 3-DOF rotational parallel manipulators. IEEE Transactions on Robotics and Automation, 2004, 20(1): 117-121.

[68] Y F Fang, LW Tsai. Analytical identification of limb structures for translational parallel manipulators. Journal of Robotic Systems, 2004, 21(5): 209-218.

[69] S Guo, Y F Fang, H B Qu. Type synthesis of 4-DOF nonoverconstrained parallel mechanisms based on screw theory. Robotica, 2012, 30(1): 31-37.

[70] XW Kong, C M Gosselin. Type synthesis of 3-DOF spherical parallel manipulators based on screw theory. Journal of Mechanical Design, 2004, 126(1): 101-108.

[71] XW Kong, C M Gosselin. Type synthesis of 3-DOF translational parallel manipulators based on screw theory. Journal of Mechanical Design, 2004, 126(1): 83-92

[72] XW Kong, C M Gosselin. Type synthesis of 3T1R 4-DOF parallel manipulators based on screw theory. IEEE Transactions on Robotics and Automation, 2004, 20(2): 181-190.

[73] XW Kong, C M Gosselin. Type synthesis of three-degree-of-freedom spherical parallel manipulators. The International Journal of Robotics Research, 2004, 23(3): 237-245.

[74] XW Kong, C M Gosselin. Type synthesis of parallel mechanisms. Berlin: Springer, 2007.

[75] XW Kong, C M Gosselin. Type synthesis of 3-DOF PPR-equivalent parallel manipulators based on screw theory and the concept of virtual chain. Journal of Mechanical Design, 2005, 127(6): 1113-1121.

[76] XW Kong, C M Gosselin. Type synthesis of 5-DOF parallel manipulators based on screw theory. Journal of Robotic Systems, 2005, 22(10): 535-547.

[77] XW Kong, C M Gosselin. Type synthesis of three-DOF up-equivalent parallel manipulators using a virtual-chain approach. In: J Lennarčič, $B$ Roth. Advances in robot kinematics. Dordrecht: Springer, 2006: 123-132.

[78] XW Kong, C M Gosselin. Type synthesis of 4-DOF SP-equivalent parallel manipulators: A virtual chain approach. Mechanism and Machine Theory, 2006, 41(11): 1306-1319.

[79] W Ye, Y F Fang, S Guo, et al. Type synthesis of 2R2T parallel mechanisms based on motion equivalent chain method. Proceedings of the Institution of Mechanical Engineers, Part C: Journal of Mechanical Engineering Science, 2014, 228(17): 3209-3217.

[80] F G Xie, X J Liu, T M Li. Type synthesis and typical application of 1T2Rtype parallel robotic mechanisms. Mathematical Problems in Engineering, 2013, 2013: 1-12.

[81] F G Xie, X J Liu, Z You, et al. Type synthesis of 2T1R-type parallel kinematic mechanisms and the application in manufacturing. Robotics and Computer-Integrated Manufacturing, 2014, 30(1): 1-10.

[82] F G Xie, X J Liu, C Wang. Design of a novel 3-DoF parallel kinematic mechanism: type synthesis and kinematic optimization. Robotica, 2015, 33(3): 622-637.

[83] F G Xie, T M Li, X J Liu. Type synthesis of 4-DOF parallel kinematic mechanisms based on Grassmann line geometry and atlas method. Chinese Journal of Mechanical Engineering, 2013, 26(6): 1073-1081.

[84] F G Xie, X J Liu. Design and development of a high-speed and highrotation robot with four identical arms and a single platform. Journal of Mechanisms and Robotics, 2015, 7(4):041015.
[85] C H Kuo, J S Dai. Task-oriented structure synthesis of a class of parallel manipulators using motion constraint generator. Mechanism and Machine Theory, 2013, 70: 394-406.

[86] B Siciliano. The Tricept robot: Inverse kinematics, manipulability analysis and closed-loop direct kinematics algorithm. Robotica, 1999, 17(4): 437-445.

[87] D Zhang, C M Gosselin. Kinetostatic modeling of N-DOF parallel mechanisms with a passive constraining leg and prismatic actuators. Journal of Mechanical Design, 2001, 123(3): 375-381.

[88] D Zhang, C M Gosselin. Kinetostatic modeling of parallel mechanisms with a passive constraining leg and revolute actuators. Mechanism and Machine Theory, 2002, 37(6): 599-617.

[89] Y Lu, B Hu. Analyzing kinematics and solving active/constrained forces of a 3SPU+UPR parallel manipulator. Mechanism and Machine Theory, 2007, 42(10): 1298-1313.

[90] LW Tsai. The enumeration of a class of three-DOF parallel manipulators. Proceedings of the 10th World Congress on the Theory of Machine and Mechanisms, Oulu, Finland, June 20-24, 1999: 1121-1126.

[91] Y Lu, T Leinonen. Type synthesis of unified planar-spatial mechanisms by systematic linkage and topology matrix-graph technique. Mechanism and Machine Theory, 2005, 40(10): 1145-1163.

[92] Y Lu, L Ding, J Yu. Autoderivation of topological graphs for type synthesis of planar 3DOF parallel mechanisms. Journal of Mechanisms and Robotics, 2010, 2(1): 011002

[93] Y Lu, Y Wang, L Ding. Type synthesis of four-degree-of-freedom parallel mechanisms using valid arrays and topological graphs with digits. Proceedings of the Institution of Mechanical Engineers, Part C: Journal of Mechanical Engineering Science, 2014, 228(16): 3039-3053.

[94] X D Meng, F Gao, S F Wu, et al. Type synthesis of parallel robotic mechanisms: framework and brief review. Mechanism and Machine Theory, 2014, 78: 177-186.

[95] Z Huang, J F Liu, Y W Li. On the degrees of freedom of mechanisms. Beijing: Science Press, 2011. (in Chinese)

[96] Z Huang, Z M Chen, J F Liu, et al. A 3DOF rotational parallel manipulator without intersecting axes. Journal of Mechanisms and Robotics, 2011 3(2): 021014.

[97] Z M Chen, W A Cao, Z Huang. Type synthesis of 3-DOF rotational parallel mechanisms with no intersecting axes. Proceedings of the ASME 2012 International Design Engineering Technical Conferences and Computers and Information in Engineering Conference, Chicago, USA, August 12-15, 2012: 565-572.

[98] J J Yu, J S Dai, S S Bi, et al. Numeration and type synthesis of 3-DOF orthogonal translational parallel manipulators. Progress in Natural Science, 2008, 18(5): 563-574.

[99] J J Yu, J S Dai, S S Bi, et al. Type synthesis of a class of spatial lowermobility parallel mechanisms with orthogonal arrangement based on Lie group enumeration. Science China Technological Sciences, 2010, 53(2): 388-404.

[100] Q C Li, J M Hervé. Structural shakiness of nonoverconstrained translational parallel mechanisms with identical limbs. IEEE Transactions on Robotics, 2009, 25(1): 25-36.

[101] X J Liu, J S Wang, G Pritschow. A new family of spatial 3-DoF fullyparallel manipulators with high rotational capability. Mechanism and Machine Theory, 2005, 40(4): 475-494.

[102] C Z Wang, Y F Fang, S Guo. Design and analysis of 3R2T and 3R3T parallel mechanisms with high rotational capability. Journal of Mechanisms and Robotics, 2016, 8(1): 011004.

[103] C Z Wang, Y F Fang, H R Fang. Novel 2R3T and 2R2T parallel mechanisms with high rotational capability. Robotica, 2017, 35(2): 401-418. 